\title{
Hydrogen Plasma Phase Diagram and Properties
}

\author{
W. Ebeling, Berlin; W.D. Kraeft, Greifswald and D. Kremp, Rostock
}

(Humboldt-Universität, Ernst-Moritz-Arndt-Universität and Wilhelm-Pieck-Universität)

Hydrogen is not only the most simple element and the most abundant but also in some respects the most exotic. Here we are reporting on just the fluid plasma phases of hydrogen including the liquid metallic phase. Of special interest is the possibility of macroscopic quantum effects as e.g. superfluidity and superconductance.

The simplest hydrogen system consisting of one electron and one proton only, comprises bound states of the atom:

$$
\mathrm{e}^{-}+\mathrm{p}^{+} \rightleftharpoons \mathrm{H}
$$

while a four particle system comprises atomic, ionic as well as molecular states:

$$
\begin{aligned}
& \rightleftharpoons 2 \mathrm{H} \\
& 2 \mathrm{e}^{-}+2 \mathrm{p}^{+}\left\{\begin{array}{l}
\rightleftharpoons \mathrm{H}^{+}+\mathrm{e}^{-}+\mathrm{H} \\
\rightleftharpoons \mathrm{H}_{2}^{+}+\mathrm{e}^{-}
\end{array}\right. \\
& \rightleftharpoons \mathrm{H}_{2}
\end{aligned}
$$

In other words we have different reaction channels. In many particle systems all the reactions mentioned above may occur, modified and influenced by many particle, temperature or pressure effects ${ }^{1}$ ). The manifold of possible behaviours of hydrogen systems as a function of temperature, pressure or kinetic conditions is thus quite extensive ${ }^{2}$ ).

The existence of a metallic state of hydrogen matter was first postulated by Wigner and Huntington in 1935. Since then, many authors ${ }^{3-5}$ ) have discussed the properties of liquid metallic hydrogen including the possibility of macroscopic quantum effects, as e.g. super-

Fig. 1 - Phase diagram of hydrogen and the adiabate of Jupiter (dash-dotted) and Sun (dashed).

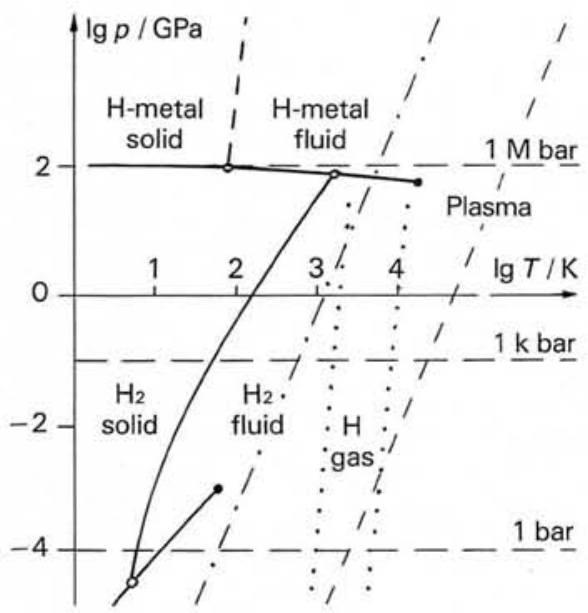

conductance. We should mention also the interesting experimental investigations in which the conditions close to the required temperatures and densities have been approached ${ }^{3}$ ). However, one must say that both the experimental and theoretical work in this field are still far from providing solutions to the many problems that are open. One of the most important questions is the metastability of high pressure phases, with the theoretical possibility of having metastable phases of hydrogen with unusual physical properties even at normal pressures and temperatures. This could of course have very important technical applications as e.g. high-temperature superconductors.

\section{Fluid Phases of Hydrogen}

In spite of the simplicity in composition, hydrogen systems have a great variety of possible stable phases. The phase diagram of hydrogen, which is still hypothetical in the high-pressure region, is shown in Fig. 1. Let us consider some isobars and isotherms, beginning with the isobar $p=10^{3}$ bar $\left(10^{8} \mathrm{~Pa}\right)$. Coming from the high-temperature side, we are first in the plasma region; then at about $10^{4} \mathrm{~K}$ we come to the narrow region of atomic hydrogen which, below $10^{3} \mathrm{~K}$, gives place to a molecular region. Finally, with decreasing temperature the hydrogen crystallizes and forms a $\mathrm{H}_{2}$ solid. At pressures below

$$
p_{\mathrm{c}}=1.3 \mathrm{MPa}\left(T_{\mathrm{c}}=33 \mathrm{~K}\right)
$$

a line of coexistence between a molecular liquid and gas phase is observed. In other words at a pressure of, e.g. $p \approx 10^{5} \mathrm{~Pa}$ ( 1 bar), the system moves with decreasing temperature through a state with two coexisting fluid phases. Below the triple point the hydrogen system goes directly from the $\mathrm{H}_{2}$ gas phase to the $\mathrm{H}_{2}$ solid phase.

Let us now consider an isotherm at say $T=1 \mathrm{~K}$. Starting with low pressures, the system is in the $\mathrm{H}_{2}$ solid state. With increasing pressure the solid breaks down at about $p=10^{11}-10^{12} \mathrm{~Pa}$ (1-10 Mbar) and goes to the hypothetical metallic hydrogen solid. The precise transition pressure is not known. However several estimates yield values in the Megabar region. For isotherms between $T_{\mathrm{t}}=1700 \mathrm{~K}\left(p_{\mathrm{t}}=230 \mathrm{GPa}\right)$,

which is the estimate for the second triple point of hydrogen after Kerley (1972), and

$$
T_{\mathrm{c}}=16500 \mathrm{~K}\left(p_{\mathrm{c}}=23 \mathrm{GPa}\right) \text {, }
$$

which is the estimate for the second critical point of hydrogen ${ }^{2}$ ), (see also ref. 4), another (hypothetical) phase transition is expected. This is the socalled plasma phase transition discussed first by Norman and Starostin (1968), and by Ebeling, Kraeft and Kremp (1976). Along the coexistence line, the plasma splits into two phases with different degrees of ionization: a highly ionized phase and a weakly ionized phase, see below.

Fig. 2 - Physical states in the density temperature diagram (dotted line: $\Lambda_{e}=d$; dash-dotted: $I=d$ ).

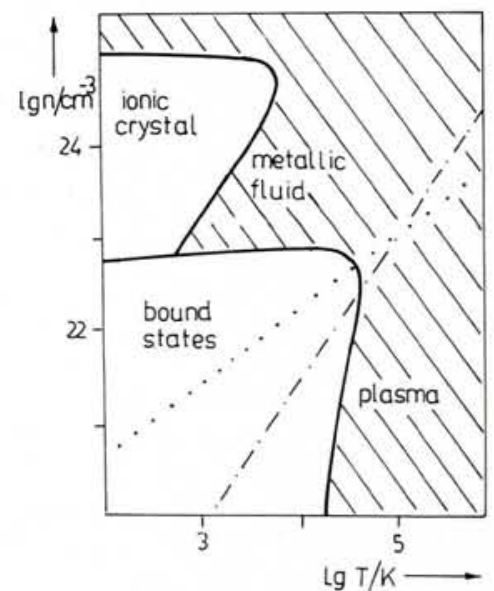

Another approach to discussing the phases of hydrogen makes reference to the density-temperature plane. Here it is useful to start from a consideration of the characteristic lengths of the system - see panel.

$a_{\mathrm{e}}=0.5292 \times 10^{-10} \mathrm{~m}-$

electronic Bohr radius,

$a_{\mathrm{p}}=0.2882 \times 10^{-15} \mathrm{~m}-$

protonic Bohr radius

$d=\left(3 / 4 \pi n_{p}\right)^{1 / 3}-$

mean distance between protons

$\Lambda_{e}=h\left(2 \pi m_{\mathrm{e}} k_{\mathrm{B}} T\right)^{-1 / 2}-$

thermal De Broglie wave length of electrons

$\Lambda_{\mathrm{p}}$ - corresponding quantity for protons

$I=e^{2} / k_{\mathrm{B}} T$ - Landau length

The border of strong coupling is given by:

$$
l \cong d
$$

and the borders of degeneracy by

$$
\Lambda_{\mathrm{e}} \cong d \text { and } \Lambda_{\mathrm{p}} \cong d \text {. }
$$


At $d \leqq 10^{2} /$ the protons may form a Wigner lattice. At $d \leq a_{p}$ the protons behave like an ideal Fermi fluid, and at $d \leq a_{\mathrm{e}}$ the electrons may be considered to be ideal. Different regions corresponding to this classification are shown in Fig. 2.

Understanding the physical properties of many particle hydrogen systems is not just of inherent interest but also of practical significance. In the first place there are direct astrophysical applications ${ }^{3,4}$ ). With a level of about $70 \%$, hydrogen is the most abundant of the elements and to a certain approximation the early stages of the evolution of our Universe from an age of say 10 minutes to 1 million years corresponded to a hot hydrogen-helium plasma which was adiabatically expanding. In other words, the Universe moved along an adiabate, crossing at about $10^{5}$ years after the big bang the conditions for the formation of bound states (atoms and molecules). The interior of stars and the giant planets covers a wide range of states as can be seen in Fig. 1 where two adiabates are plotted, corresponding to the states along a radial section of Jupiter and the Sun. In the centre of the giant planets are conditions (pressures up to $35 \times 10^{16} \mathrm{~Pa}$ and temperatures up to $20000 \mathrm{~K})$ which are in the region of a liquid metallic state ${ }^{4}$ ).

\section{Effective Interaction and Bound States ${ }^{1,6}$ )}

From the point of view of quantum mechanics, the behaviour of an isolated e-p pair is described by the Schrödinger equation, the solutions of which are well known. For positive energies we have a scattering state solution, i.e., the probability is finite even for very large separation of the electron and the proton. For the bound state solutions, which correspond to negative energy eigenvalues, the probability is zero to find the proton very far from the electron $(\mathrm{H}$ atom). On adding certain energy, the bound $\mathrm{H}$-atom may be transferred into a scattering state (ionization) as shown in Fig. 3.

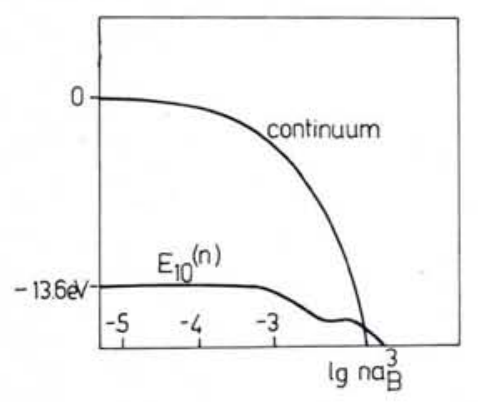

Fig. 3 - The density dependence of the continuum edge and of the bound states.

\section{Universiteit van Amsterdam}

The University of Amsterdam has an opening for a

\section{professor of condensed matter physics $(\mathrm{m} / \mathrm{f})$ *}

Full-time.

Vacancynumber E.N. 0823.

The appointed candidate's research program will constitute part of the research program of the Natuurkundig Laboratorium. This laboratory is involved in a broad spectrum of research projects in condensed matter physics.

The candidate should lead a vigorous research program and should be able to obtain funding from external sciencesupporting agencies.

Areas of research which have priority are metal physics, magnetism and semiconductor physics. It is desirable that the research program of the appointed candidate is linked to modern developments in material science. The Natuurkundig Laboratorium has experimental facilities for performing experiments at low temperatures, at high magnetic fields and at high pressures. Furthermore there is equipment to measure magnetic, thermal and optical properties, and there is an extended facility for growing single (metal) crystals.

Gross salary depends on age and experience and ranges from Dfl. 6,854.- to Dfl. 9,495.- per month (Dutch Civil Servants Code).

Applicants are requested, quoting vacancynumber, to send a curriculum vitae, list of publications and the names and addresses of a few referees, willing to provide information concerning personal and scientific qualifications - within four weeks after date of publication - to Prof. Dr. A. Lagendijk, Natuurkundig Laboratorium, Valckenierstraat 65, 1018 XE Amsterdam, The Netherlands.

Information concerning the position can be obtained from the Chairman of the Search Committee Prof. Dr. A. Lagendijk, telephone 020-522 2716.

Those wishing to draw attention to potential candidates are welcome to contact the Committee Chairman.

*Women in particular are invited to apply. 
a)

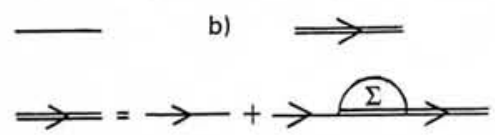

d)<smiles>[V]C1(I)CCI(I)I1I</smiles>

e)

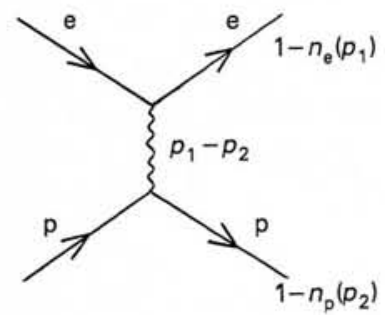

f)
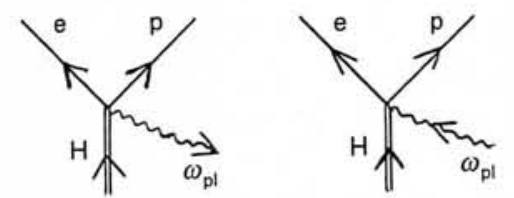

Fig. 4 - Feynman diagrams.

Entirely new aspects must be considered if the e-p pair interacts with the surrounding medium (plasma) and the problem becomes rather complicated. The behaviour of the $\mathrm{H}$-atom is modified by three effects:

The first is the self energy, which takes into account the modifications arising from the propagation of a single $\mathrm{e}$ or $\mathrm{p}$ through the plasma. This is illustrated in Fig. 4 where the propagation of a free particle is represented by an arrow (Fig. 4a), and that of an interacting one by a fat arrow (Fig. 4b). This may be expressed by the relation of Fig. $4 \mathrm{c}$ where the interaction of the $e(p)$ with the plasma is represented by the self energy

Fig. 5 - The equation of state in the high pressure region. Theoretical estimates $\left.{ }^{2}\right)$ for the metallic liquid in comparison with several points obtained from adiabatic compression experiments ${ }^{3}$ ).

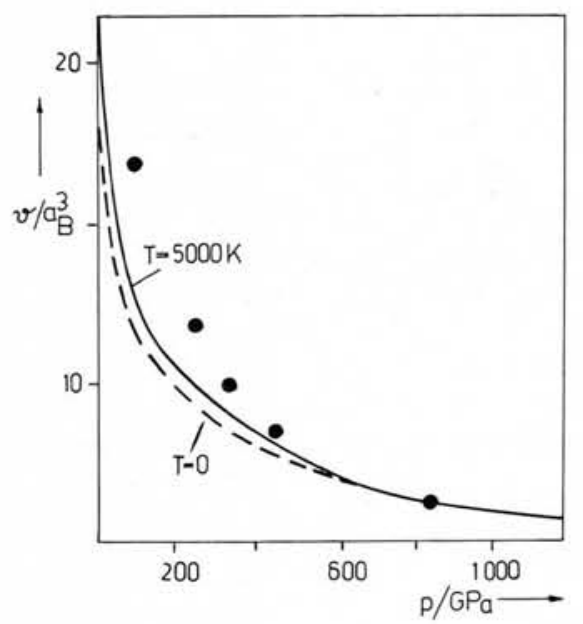

Fig. 6-Degree of ionization as a function of the density.

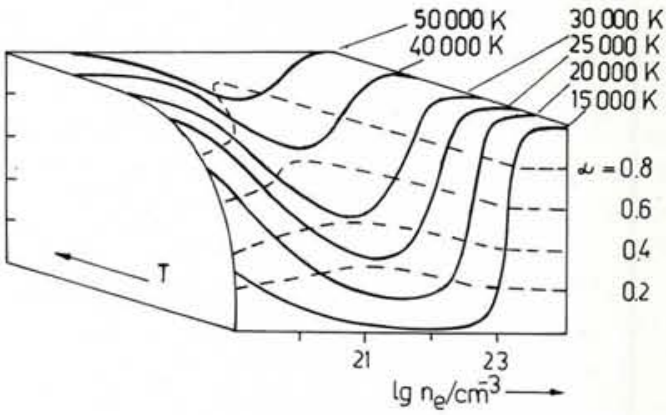

$\Sigma$. Obviously the behaviour of a pair is in fluenced by the self energy and we may say that we have to deal with the bound state problems of quasi particles, which are, separately, at zero momentum in an energy valley $(E<0)$.

A second typical effect is the screening of the long range Coulomb potential by the surrounding plasma, so that in reality, the e-p pair in a plasma interacts via a screened potential, which in the language of Feynman diagrams may be represented as in Fig. 4d. The screened potential is thus essentially determined by the polarization $\Pi$ of the plasma.

The last essential effect is the socalled Pauli-blocking, according to which the interaction between $e$ and $p$ is possible only if the final states are not occupied. Symbolically this may be represented as in Fig. $4 \mathrm{e}$ where $n_{\mathrm{e}}\left(p_{1}\right)$ is the Fermi distribution. Taking into account the three processes mentioned above, we may derive an effective Schrödinger equation which describes the behaviour of the e-p pair, and especially the modification of the properties of the $\mathrm{H}$-atom, by the interaction with the plasma. It takes into account many body effects through density and temperature-dependent terms. For example the potential appears always in connection with "blocking factors" containing the Fermi distribution.

An approximation of physical interest is the case in which the effective interaction describes the decay of an $\mathrm{H}$-atom into a free e-p pair under stimulated emission of a plasmon with frequency $\omega_{p l}$ and the inverse process, in which a plasmon is absorbed, and the final state is again a free one (Fig. 4f).

If we consider the correction terms in the effective Schrödinger equation to be a perturbation, the energy eigenvalues of the e-p pair in the plasma may be determined. The results are represented in Fig. 3. We see that the ground state is only weakly density dependent whereas in contrast, the continuum edge, i.e. the energy of scattering states for $p_{1}=p_{2}=0$, is strongly density dependent on account of the density dependence of the self energy.
Obviously the difference between the ground state energy and the continuum edge is the ionization energy. From Fig. 3 it follows that this decreases with increasing density, the cross-over between the ground state energy and the continuum edge defining the Mott density $n_{\text {Mott }}$. At higher densities, $n>n_{\text {Mott' }}$ bound states do not exist, an effect that turns out to be essential for understanding the behaviour of matter at high pressures, and it determines crucially the thermodynamic and transport properties of $\mathrm{H}$-plasmas.

\section{Equation of State and Ionization \\ Equilibrium}

Fully ionized fluid phases may be considered to be a mixture of two Fermi fluids. Fig. 5 shows the result of theoretical estimates for the high pressure region of the equation of state ${ }^{2}$ ) in comparison with experimental results ${ }^{3}$ ). The highest pressures reached are still below $10^{12} \mathrm{~Pa}$ and the results are loaded with uncertainties. One may hope that new experiments which are under way will shed new light on this question.

For regions with bound states, the thermodynamic description is much more complicated. In this case, e.g., the pressure is determined by all contributions of elementary and composite particles and the scattering between them. Restricting our considerations to fluid phases only, the equation of state must cover the partially ionized plasma and the neutral liquid and gas as well. Such equations were considered in $1,2,4)$. In addition one must use an equation for the plasma composition, i.e., a mass action law, or Saha equation.

For the single reaction:

$$
\mathrm{e}^{-}+\mathrm{p}^{+} \rightleftharpoons \mathrm{H}
$$

the balance equation for the densities reads:

$$
n_{\mathrm{p}}=n_{\mathrm{e}}=n_{\mathrm{e}}^{*}+n_{\mathrm{H}}^{*}, n_{\mathrm{e}}^{*}=n_{\mathrm{p}}^{*}
$$

where the stars denote the densities of free species. Chemical equilibrium conditions yield the Saha equation

$$
n_{\mathrm{H}}^{*} / n_{\mathrm{e}}^{*} n_{\mathrm{p}}^{*}=\Lambda_{\mathrm{ep}}^{3} \exp \left(/^{\text {eff }} / k_{\mathrm{B}} T\right)
$$

$\left(\Lambda_{\text {ep }}=h\left(2 \pi\left(m_{\mathrm{e}}+m_{\mathrm{p}}\right) k_{\mathrm{B}} T\right)^{-1 / 2}\right.$, thermal wavelength). Corresponding equations are valid for other reactions such as: 


$$
\mathrm{H}+\mathrm{H} \rightleftharpoons \mathrm{H}_{2} \text {. }
$$

Mass action laws give the composition of the partially ionized plasma, while the effective ionization energy, $l^{\text {eff }}$, must be determined from statistical theory 1,2).

From the discussion of the bound state problem and the relevant effective interaction we know that at a certain density all bound states vanish. This Mott effect for the two particle bound state energies leads to a steep increase in the degree of ionization, i.e., it changes the composition of the plasma drastically. This effect is referred to as the Mott transition and may be seen in Fig. 6, where we show the degree of ionization as a function of the density for different temperatures.

With an equation of state and the plasma composition we may determine the pressure or other thermodynamic quantities as a function of density and temperature for a partially ionized plasma.

\section{Plasma Phase Transition ${ }^{1,2}$ )}

Of high interest in the question of phase transitions, as noted above. One has, e.g., to investigate the violation of the stability conditions $\partial p / \partial V \leq 0$ which signals the possibility of phase transitions of first order. Alternatively we may consider the chemical potential of the plasma, $\mu$, when the corresponding stability condition is:

$$
\partial \mu / \partial n_{\mathrm{e}} \geq 0, n_{\mathrm{e}}=N_{\mathrm{e}} / V .
$$

Fig. 7 shows an example, where we find a violation of the stability condition. We observe a typical van-der-Waals-like behaviour, signalling the possibility of a phase transition of first order. This phase transition is the plasma phase transition discussed at the beginning. In the vicinity of the critical point it is essentially determined by the Coulomb interaction and the ionization equilibrium. At lower temperatures the interaction between neutral particles is important.

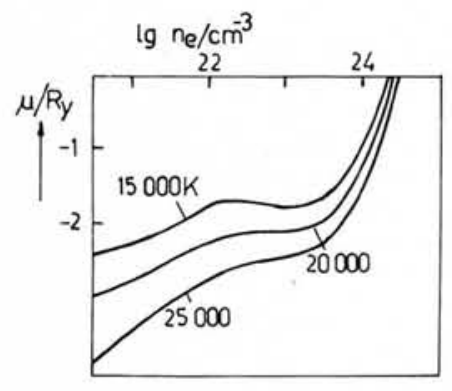

Fig. 7-Chemical potential as a function of the density.
Fig. 8 - The line of coexistence for the plasma phase transition.

$A$ - fluid molecular $\mathrm{H}$

$B$ - weakly ionized $H$

C - strongly ionized $\mathrm{H}$

$t_{2}$ - estimate of the second triple point

$\mathrm{C}_{2}, \mathrm{C}_{2}^{\prime}$ - estimate of the second critical point in two approximations

$D$ - liquid metal

E - molecular solid.

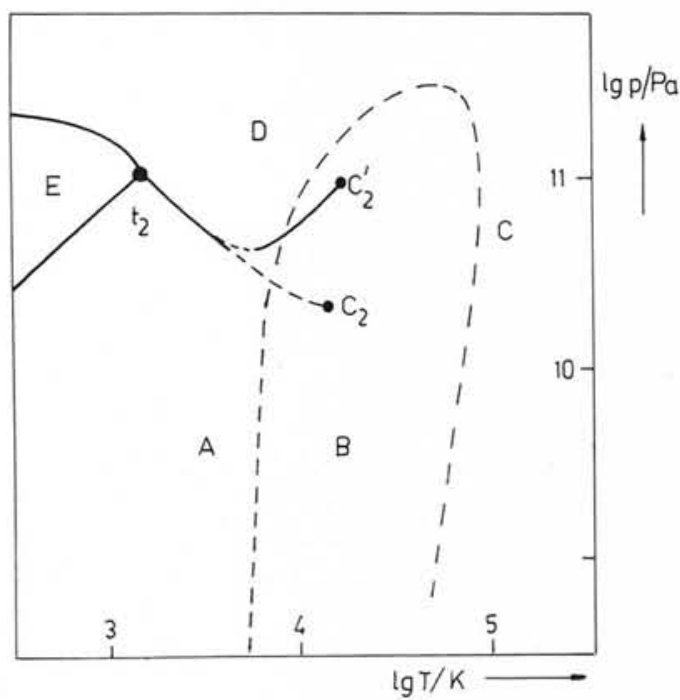

The main feature of the phase transition is the coexistence of two phases having different degrees of ionization. The low density phase is a partially ionized plasma reaching from a highly ionized plasma to a molecular fluid. The high density phase is the metallic liquid.

The corresponding coexistence curve is indicated in Fig. 1 and is shown in more detail in Fig. 8. A phase transition of such a type is predicted by the theory and may be referred to as a Mott phase transition although experimentally such a transition has (at least for hydrogen), not been detected yet; experiments ${ }^{3}$ ) do not cover the relevant area completely.

\section{REFERENCES}

1. Kraeft W.D. et al., Quantum Statistics of Charged Particles, (Akademie-Verlag, Berlin) 1985; (Plenum Press, N.Y.) 1985.

2. Schlanges M. and Kremp D., Ann. Physik 39 (1982) 69; Phys. Lett. 110A (1985); Ebeling W. and Richert W., Phys. Lett. 108A (1985) 80; Physica 130A (1985) 587.

3. Grigoriev F.V. et al. Sov. Phys JETP 69 (1975) 743; Nellis A. et al., Phys. Rev. A 27 (1983) 608 .

4. Robnik M. and Kundt W., Astron. Astrophys. 120 (1983) 227.

5. Carlson A.E. and Ashcroft N.W., Phys. Rev. B 29 (1984) 479; Campbell C.E. and Zabolitzky J.G., Phys. Rev. B 29 (1984) 123. 6. Zimmermann R., Kilimann K., Kraeft W.D., Kremp D. and Röpke G., Phys. Stat. Sol. (b) 90 (1978) 175.

\section{Kernfysisch Versneller Instituut,

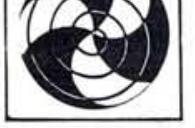 \\ Groningen The Netherlands \\ Staff Position in \\ Experimental Nuclear Physics}

The KVI wishes to appoint a young experimental nuclear physicist on a tenured staff position. The appointment will be with FOM, the Netherlands' Foundation for Fundamental Research on Matter.

The Kernfysisch Versneller Instituut is a national research institute in nuclear physics, jointly sponsored by the University of Groningen and by FOM. It has a broad experimental and theoretical research program on nuclear structure and heavy-ion physics.

At present the main facility of the $\mathrm{KVI}$ is a $\mathrm{K}=160 \mathrm{MeV}$ AVF-cyclotron for the acceleration of light and heavy ions. A large variety of detectors such as a magnetic spectrograph and various systems for the detection of light and heavy ions, electrons and $\gamma$-radiation is available. Recently funds have been made available for the design and construction of a $K=600$ MeV superconducting cyclotron for light and heavy ions. The machine which will be built in Orsay in close collaboration with the Institut de Physique Nucléaire, will replace the present $\mathrm{K}=160 \mathrm{MeV}$ cyclotron at the KVI in 1991.

The successful candidate should be interested in the research that can be performed with the existing and future facility, and should also be willing to take on responsibilities for new instrumental developments. Several years of post-doctoral experience are required.

Applicants for the above-mentioned position are requested to submit a curriculum vitae, list of publications and the names and addresses of three references, as early as possible but not later than 15 May 1986, to:

Prof. dr. R.H. Siemssen

Kernfysisch Versneller Instituut, Zernikelaan 25 NL - 9747 AA Groningen, The Netherlands 